\title{
Relações entre gestão do conhecimento, aprendizagem organizacional e educação corporativa
}

\author{
Celi Langhi ${ }^{1}$ \\ Denilson de Sousa Cordeiro 2
}

\begin{abstract}
RESUMO
Empresas brasileiras têm exigido novas competências de seus colaboradores. Para isso, novos modelos de gestão, aprendizagem e educação têm sido adotados, a fim de harmonizar os interesses das pessoas e suas carreiras com os objetivos estratégicos das corporações. Este artigo tem o objetivo de identificar relações entre gestão do conhecimento, aprendizagem organizacional e educação corporativa. A partir da abordagem dos conceitos em textos acadêmicos produzidos sobre os tópicos, os assuntos foram articulados de modo a trazer maior compreensão sobre o tema. Como resultado, foi possível analisar como o conhecimento é obtido, armazenado, disseminado e empregado pelas empresas, o que fomenta a aprendizagem no ambiente corporativo, viabilizando o planejamento e a implantação de programas de educação que não apenas qualificam os colaboradores, mas também os ajudam a exercer a liderança nos diversos grupos de trabalho das organizações, o que traz diversos benefícios para indivíduos, grupos, empresas, comunidades e para a sociedade.
\end{abstract}

PALAVRAS-CHAVE: Gestão do conhecimento. Aprendizagem Organizacional. Educação corporativa.

\footnotetext{
${ }^{1}$ Doutora em Psicologia Escolar e do Desenvolvimento Humano pelo Instituto de Psicologia da Universidade de São Paulo, São Paulo, SP, Brasil. Orcid: https://orcid.org/0000-0002-5527-2412.E-mail: celi@infolearning.com.br.

${ }^{2}$ Mestrando do Programa de Mestrado Profissional em Gestão e Desenvolvimento da Educação Profissional. Centro Paula Souza, São Paulo, SP, Brasil. Orcid: https://orcid.org/0000-0003-4686-2477. E-mail: denilson.cordeiro@cpspos.sp.gov.br.
} 


\title{
Relationships between Knowledge Management, Organizational Learning and Corporate Education
}

\begin{abstract}
Brazilian companies have demanded new skills from their employees. To this end, new models of management, learning and education have been adopted, in order to harmonize the interests of people and their careers with the strategic objectives of corporations. This article aims to identify relationships between knowledge management, organizational learning and corporate education. From the approach of the concepts in academic texts produced on the topics, the subjects were articulated in order to bring greater understanding on the theme. As a result, it was possible to analyze how knowledge is obtained, stored, disseminated and used by companies, which promotes learning in the corporate environment, enabling the planning and implementation of education programs that not only qualify employees, but also help them to exercise leadership in the various working groups of organizations, which brings several benefits to individuals, groups, companies, communities and to society.
\end{abstract}

KEYWORDS: Knowledge management. Organizational Learning. Corporative education.

Relaciones entre la Gestión del Conocimiento, el Aprendizaje Organizacional y la Educación Corporativa

\section{RESUMEN}

Las empresas brasileñas han exigido nuevas habilidades a sus empleados. Para ello, se han adoptado nuevos modelos de gestión, aprendizaje y educación, con el fin de armonizar los intereses de las personas y sus carreras con los objetivos estratégicos de las corporaciones. Este artículo tiene como objetivo identificar las relaciones entre la gestión del conocimiento, el aprendizaje organizacional y la educación corporativa. A partir del abordaje de los conceptos en los textos académicos producidos sobre los temas, se articularon las asignaturas con el fin de aportar una mayor comprensión sobre el tema. Como resultado, fue posible analizar cómo el conocimiento es obtenido, almacenado, difundido y utilizado por las 
empresas, lo que promueve el aprendizaje en el entorno corporativo, posibilitando la planificación e implementación de programas educativos que no solo capacitan a los empleados, sino que también los ayudan. Ejercer el liderazgo en los distintos grupos de trabajo de las organizaciones, lo que aporta diversos beneficios a las personas, grupos, empresas, comunidades y la sociedad.

PALABRAS CLAVE: Conocimiento administrativo. Aprendizaje organizacional. Educación corporativa.

$$
* * *
$$

\section{Introdução}

As novas estruturas organizacionais das empresas brasileiras exigem cada vez mais do desempenho de seus profissionais contratados, no tocante a agilidade, flexibilidade, competências sociais, pró-atividade e criatividade. Isto se reflete na área de treinamento e desenvolvimento (T\&D), na qual alguns conceitos relacionados à aprendizagem humana revelam novas perspectivas a respeito de conceitos como informação, instrução, treinamento, desenvolvimento e educação (ESTEVES; MEIRIÑO, 2015).

Considerando que as pessoas são capazes de aprender por meio de várias maneiras (leitura, observação, pesquisa, conversa, viagem, sala de aula, meios virtuais, etc.), entende-se que a renovação nas áreas de T\&D e de gestão (de pessoas e de conhecimento) é indispensável, uma vez que não basta dar treinamentos aos indivíduos. Assim, surgem novas maneiras de pensar e trabalhar, para que indivíduos venham a desenvolver suas habilidades e competências por meio de programas de ensino e de aprendizagem nas organizações, nos quais o aprendizado contínuo demanda o incremento de qualificações mais abrangentes. Isto leva ao crescente comprometimento das empresas com aprendizagem e educação (ESTEVES; MEIRIÑO, 2015).

Tendo como ponto de partida esse aumento pelo interesse no tratamento do conhecimento nas empresas, este artigo se propõe a identificar relações entre os conceitos de gestão do conhecimento, aprendizagem organizacional e educação 
corporativa. Para isto, serão abordados os conceitos já citados, analisando de que modo se articulam nas práticas sociais adotadas pelas empresas que demonstram foco nos resultados dos objetivos estratégicos organizacionais a partir do investimento nas competências de seus colaboradores.

\section{Metodologia}

O trabalho realizado a partir da leitura e revisão de outros trabalhos já desenvolvidos, com teor analítico, é valorizado por contribuir com o desenvolvimento do conhecimento científico, pois se presta a compactar e comparar o conhecimento presente em fontes diversas. Ele aponta para a existência de possíveis especializações e áreas de interesse, direcionando a realização de futuras pesquisas, além de servir de fonte de consulta tanto para estudiosos experientes quanto para iniciantes do mundo acadêmico (ANTONELLO; GODOY, 2009).

Considerando a proposta inicial deste artigo - identificar relações entre gestão do conhecimento, aprendizagem organizacional e educação corporativa -, foi realizada uma pesquisa bibliográfica, a fim de delinear de forma mais específica os conceitos que compunham o objeto da pesquisa, com a finalidade de apropriação de conhecimentos para compreensão mais profunda dos assuntos (TOZONI-REIS, 2009).

A revisão descritiva, com o objetivo de resumir conhecimentos prévios, foi a estratégia metodológica adotada para a revisão de literatura, a fim de apoiar ou revelar estruturas e tendências a respeito das proposições e teorias pré-existentes (PARÉ et al., 2015). A coleta de dados qualitativos, a organização dos referidos dados, sua análise e interpretação foram as etapas pelas quais a produção textual percorreu. Isto resultou na elaboração da discussão e das considerações sobre as relações entre as partes investigadas. 
Desta forma, procurou-se propor objetivos que contribuem com a produção textual e o estudo dos textos acadêmicos selecionados, com esforços empreendidos para a sistematização do trabalho de leitura, interpretação e discussão.

\section{Gestão do conhecimento}

As inovações dependem de níveis pré-existentes de conhecimento e de entendimento. A maior fonte de conhecimento se encontra fora dos limites de uma organização corporativa, e para acessar o conhecimento externo é imprescindível que a empresa tenha a capacidade de localizá-lo, compreendêlo, assimilá-lo e internalizá-lo (PATON; PETERS; QUINTAS, 2007).

A gestão do conhecimento $(\mathrm{GC})$ tem sido vista como um recurso das organizações para manter sua competitividade. É um processo que viabiliza o fluxo do conhecimento entre pessoas e grupos nas empresas, descrito como uma sequência de quatro grandes etapas: aquisição (absorção do conhecimento), armazenamento (identificação e localização do conhecimento), distribuição (aprendizagem em todos os níveis da empresa) e utilização (busca de melhores práticas) do conhecimento (NEVES; CERDEIRA, 2018).

A absorção do conhecimento pelas organizações pode se dar por meio da aprendizagem das pessoas em organizações, e pode ocorrer a partir de treinamentos formais, esquemas educacionais e programas de pesquisa e desenvolvimento. Outras modalidades de aprendizagem são decorrentes de aquisição de tecnologias, que possuem embutido o conhecimento necessário à empresa (PATON; PETERS; QUINTAS, 2007). Uma organização também pode absorver conhecimentos de profissionais contratados para compor seu quadro funcional.

Contratar especialistas como consultores que transmitam o conhecimento especializado pode ser algo eficiente para fomentar a absorção de conhecimento da organização, desde que a empresa tenha estratégias para 
maximizar a aprendizagem a partir de tais consultores. Outra maneira de viabilizar a internalização de conhecimento nas organizações é a seleção e contratação de gestores e colaboradores que possuam saberes e competências condizentes com os objetivos estratégicos organizacionais.

Os processos seletivos para contratações não se dedicam a apenas preencher vagas, mas também à adequação de profissionais ao quadro funcional da organização. Os resultados alcançados em menor prazo se apresentam na redução quantitativa de indicadores, tais como: taxa de rotatividade, taxa de absenteísmo, custos de investimentos na contratação, gastos com treinamento, tempo e custo de integração e adaptação de candidatos (ESTEVES; MEIRIÑO, 2015). Considerando esses efeitos, não basta que o conhecimento seja absorvido (adquirido) pelas empresas. É necessário também garantir o seu armazenamento.

Esforços para preservar o conhecimento existente nas organizações implicam em referenciar o conhecimento relevante e em identificar sua localização no âmbito corporativo. Não se pode criar mecanismos de obtenção e armazenamento de conhecimento se este não for localizado e identificado, sendo estes os primeiros desafios da GC. Muitas organizações não têm informações sobre a natureza e localização do conhecimento de seus colaboradores, o que dificulta consequentemente a obtenção adequada do conhecimento e sua utilização (NEVES; CERDEIRA, 2018). Superada a fase de armazenamento do processo da GC, é necessário garantir que o conhecimento seja adequadamente distribuído na organização.

Sob a lógica da GC, a aprendizagem deve ocorrer em todos os níveis de uma organização, pois isto transforma conhecimento em competências, por meio de mobilização, aprendizagem, participação e comprometimento de todos os gestores e colabores. A distribuição de conhecimento entre as pessoas da organização pode ocorrer com recursos de tecnologias de informação, bem como pela promoção de práticas de interação entre pessoas e grupos da empresa. O uso de tecnologias de comunicação para o 
armazenamento implica na codificação do conhecimento, o que pode ser realizado apenas por pessoas (NEVES; CERDEIRA, 2018).

O papel das pessoas é muito relevante na GC. Por isso, é preciso que os colaboradores das organizações partilhem por vontade própria o conhecimento, de modo que possam evidenciar o seu valor e significado para os diversos processos organizacionais. Ao passo que o conhecimento é inerente ao indivíduo, é dever da empresa apoiar colaboradores criativos para criar e transformar o conhecimento (ESTEVES; MEIRIÑO, 2015).

Com auxílio e incentivo da organização, o indivíduo pode estar sempre buscando o aprendizado contínuo para abraçar novas atribuições e responsabilidades. Competência é o termo que o qualifica a executar atividades com excelência, conhecimento, habilidade e atitudes satisfatórias ao seu desempenho. A disseminação de competências se dá no âmbito da organização, que viabiliza o compartilhamento do conhecimento através da gestão que prepare os indivíduos para novos desafios, ampliando os aspectos intelectuais, emocionais e morais de sua competência num processo que continuamente permita que o conhecimento na organização seja não apenas acessível e compartilhável, mas também utilizável (ESTEVES; MEIRIÑO, 2015).

O conjunto de conhecimento adquiridos, armazenados e distribuídos pelas organizações corporativas é uma forma de mapa com grandes quantidades de informações sobre experiências e vivências passadas, cuja utilização permite às empresas que evitem cometer os mesmos erros, assegurando a contínua busca de melhores práticas com base na sabedoria coletiva (NEVES; CERDEIRA, 2018). O emprego do conhecimento ajuda no desenvolvimento das corporações, permitindo concentrar esforços no alcance das expectativas das organizações.

O foco em resultados é uma das competências mais visadas pelas organizações corporativas. Trata-se da capacidade de cumprir objetivos e metas ao mobilizar pessoas para o alcance da visão estratégica organizacional, com a performance de atitudes criativas e eficazes para obter 
resultados, bem como com a antecipação de oportunidades de vantagem competitiva para a empresa (ESTEVES; MEIRIÑO, 2015).

A GC, portanto, é uma ferramenta que possibilita às organizações identificar, armazenar, transformar e empregar informações e conhecimentos especializados. Da necessidade do envolvimento dos colaboradores e gestores no processo de GC surgem as práticas de aprendizagem organizacional, as quais ocorrem de acordo com a dinâmica das organizações nas quais o conhecimento é construído e tomam diferentes formas em função das especificidades das empresas.

\section{Aprendizagem organizacional}

A aprendizagem organizacional (AO) pode ser conceituada como modo pelo qual empresas constroem, proveem e organizam seus conhecimentos e rotinas de atividades, dentro de suas culturas, com adaptação e desenvolvimento da eficiência organizacional através de melhorias de habilidades básicas de suas forças de trabalho (PATON; PETERS; QUINTAS, 2007). Sua finalidade é o alcance de novos, múltiplos e contínuos conhecimentos sobre dinâmicas e demandas corporativas, tanto de maneira direta como indireta, dentro e fora da organização. Isto confere um ponto de partida para algumas considerações sobre o assunto.

Via de regra, seis variáveis permitem à $\mathrm{AO}$ fomentar o desenvolvimento integrado das organizações: cultura, estrutura, foco no grupo, visão estratégica, gestão integrada dos recursos humanos e comunicação. A implementação de iniciativas destinadas a melhorarem a AO e a transformarem organizações em organizações que aprendem abarca quatro subprocessos: aquisição do conhecimento, interpretação da informação recebida, distribuição da informação e memória organizacional (SANTANA, 2005). Estes subprocessos se articulam no macroprocesso que fomenta o ensino e a aprendizagem nas empresas. 
A AO possui uma natureza processual. A perspectiva vigente sobre processos de aprendizagem valoriza a tendência a contínuas mudanças nas organizações, bem como emprega níveis de análise individual, grupal e organizacional. De acordo com Antonello e Godoy (2009, p. 278):

A AO, em sua história, foi caracterizada como incremento/melhoria de desempenho no transcorrer do tempo, como fenômeno comportamental, cognitivo, sociocultural, fenômeno com dimensões reflexivas e emocionais, fenômeno experiencial. O que se percebe, em alguns casos, é que os estudos e as teorias propostas para a $\mathrm{AO}$ pecam por não levarem em conta as lições oriundas da experiência e dos diversos estudos já desenvolvidos na área de conhecimento em aprendizagem.

Para as autoras (ANTONELLO; GODOY, 2009), existem cinco características cuja discussão precisa ser aprofundada, tanto para a compreensão do fenômeno quanto para o delineamento de um conceito de AO que embase estudos empíricos. Identificam-se os seguintes aspectos:

1. Nivel da aprendizagem: é possível considerar a aprendizagem como fenômeno interpessoal. A AO recorre tanto ao indivíduo quanto a suas interações grupais, intergrupais, organizacionais e até mesmo interorganizacionais. É necessário um motivo para separar $\mathrm{AO}$ de aprendizagem individual, bem como um mecanismo para explicar como grupos e organizações aprendem. Entretanto, pode-se também considerar a aprendizagem conectada com ambos os níveis - individual e organizacional.

2. Neutralidade da meta: aprendizagem pode ser vista como algo positivo ou negativo. Com a redefinição da relação entre $\mathrm{AO}$ e os resultados de desempenho de grupos e organizações, uma relação positiva é comumente assumida, quer seja pela definição de aprendizagem, quer seja por sua operacionalização, o que torna trivial o conceito de AO.

3. Noção de mudança: nem toda aprendizagem acarreta mudança no comportamento. Há de se separar as noções de aprendizagem e mudança organizacional (processo a que aquela é frequentemente relacionada). 
4. Natureza processual da aprendizagem: aprendizagem é processo, devendo ser estudada como tal. Com a recuperação da dimensão de processo de aprendizagem, é possível obter-se um desenvolvimento interessante do conceito de $\mathrm{AO}$ e de suas pesquisas.

5. Natureza politica da aprendizagem: é necessário considerar as relações de poder intrínsecas aos processos de AO, visto que sua prática apresenta noções complexas embutidas em estruturas sociais que envolvem relações de poder.

Considerando-se os cinco aspectos apresentados e transferindo-se a noção de processo de mudança para a realidade nas organizações, fica perceptível o surgimento de um processo que conecta atores, sistemas, atividades articuladas e artefatos. Tal perspectiva destaca que a AO não pode ser vista apenas como um processo individual, mas notada a partir das relações e interações das pessoas com os elementos sociais e materiais dos contextos particulares das organizações, suas divisões de trabalho, relações de poder, cultura, linguagem e outros elementos semelhantes (ANTONELLO; GODOY, 2009).

O ciclo de aprendizagem individual é um processo, no qual crenças individuais se modificam e são codificadas nos modelos mentais das pessoas. Esses ciclos afetam a AO mediante sua influência nos modelos mentais compartilhados entre colabores e gestores da organização. Contudo, indivíduos podem aprender à revelia da organização, podendo a aprendizagem individual ser relevante ou não para a aprendizagem da organização (SANTANA, 2005).

As partes da aprendizagem individual relevantes para a $\mathrm{AO}$ constituem a memória ativa à qual a organização presta atenção, influenciando o modo organizacional de atuar e passando a compor os modelos mentais de experiências compartilhados que tornam a memória organizacional utilizável. Dito isto, a aprendizagem individual depende da capacidade dos indivíduos melhorarem seus modelos mentais, e a explicitação deste modelos é imprescindível para o desenvolvimento de 
modelos mentais compartilhados. Este processo marca a independência da AO em relação a indivíduos específicos (SANTANA, 2005).

Deste modo, a noção de AO como processo para alcançar conhecimentos relacionados à realidade e às necessidades das organizações contribui para que a dinâmica das empresas seja compreendida, viabilizando fluxos de mudança nos sistemas sociais. O processo de aprendizagem no qual mudanças são implementadas e disseminadas nas empresas é materializado e avaliado através dos programas de educação corporativa desenvolvidos pelas organizações.

\section{Educação corporativa}

A educação corporativa (EC) é uma iniciativa organizacional, com o propósito de garantir um processo contínuo e estruturado de aprendizagem, a qual deve estar vinculada a objetivos estratégicos. É um sistema de desenvolvimento de pessoas, calcado na gestão por competências. Seu papel é conectar o aprimoramento individual à estratégia de atuação da empresa. É uma forma inteligente para que haja domínio do negócio de educação e treinamento pelas organizações, com melhor aproveitamento de recursos e vinculação de programas educacionais ao desenvolvimento das competências exigidas pelas estratégias do negócio (CRUZ, 2010).

A EC surge no Brasil no final do século XX, por meio da coordenação entre gestão de pessoas e GC como um trabalho em progresso, a fim de incluir nas estratégias organizacionais o desenvolvimento educacional dos diversos stakeholders (colaboradores, fornecedores e clientes), por meio da ampliação de competências individuais e organizacionais de capacitação (ESTEVES; MEIRIÑO, 2015), o que a diferencia dos programas de treinamentos em voga até então.

Diferente de treinamento empresarial ou qualificação de mão-de-obra, a EC se relaciona com uma nova maneira de pensar e trabalhar, "moldando a 
visão de aprendizagem contínua, fixando metas para a organização, agregando valor ao negócio” (ESTEVES; MEIRIÑO, 2015, p. 4), configurandose como um guarda-chuva estratégico para o desenvolvimento e educação de uma comunidade, com a intenção de cumprir as estratégias da organização, o que constitui um modelo com base na sociedade do conhecimento transformação do indivíduo social através do conhecimento, o que impõe inúmeros desafios à educação escolar tradicional.

Sob a perspectiva de que o Estado brasileiro não oferece mão de obra qualificada e nem formação acadêmica de excelência, as organizações corporativas assumem tal responsabilidade, dirigindo projetos educacionais, internalizando a escola nas empresas e considerando as reais expectativas dos trabalhadores. Isto confere retorno satisfatório, visto que a celeridade com que a informação e o conhecimento sofrem obsolescência. A EC viabiliza sistemas de aprendizagem que reúnem os interessados no alcance da meta empresarial - colaboradores, clientes e cadeia produtiva (ESTEVES; MEIRIÑO, 2015), fazendo com que as possibilidades de desenvolvimento individual de carreiras e interesses estejam alinhadas com a estratégia organizacional, e não com o acúmulo de qualificações profissionais.

De acordo com Esteves e Meiriño (2015), os programas de EC concorrem atualmente para que conceitos como qualificação de funções de gestores e/ou colaboradores sejam ultrapassados, haja vista que é preciso investimentos para o desenvolvimento de novos perfis para um modo inusitado de agir e pensar quando se trata dos quadros funcionais das organizações. Para que a vantagem competitiva entre empresas seja de fato alcançada a partir do desempenho e da antecipação de mudanças diferenciados, a EC deve dar maior préstimo ao capital humano, ocupando novos espaços nas organizações corporativas.

A EC não é restrita ao espaço das salas de aula. Antes, está vinculada a processos organizacionais de aprendizagem contínua e educação continuada, influenciando os recursos intelectual e pessoal das empresas, 
bem como o surgimento de novas políticas de gestão que visam ao compartilhamento de experiências, ações e informações, com o propósito de solucionar problemas, aprender a reaprender em conjunto com a equipe. Essa visão demanda do gestor/colaborador faculdades tais como: lidar com imprevistos, demonstrar pró-atividade e desenvolver o aprendizado organizacional (e não somente o individual) (ESTEVES; MEIRIÑO, 2015), como perceptíveis reflexos na capacidade de liderar pessoas.

Sobre o comportamento adequado à liderança no cenário corporativo, Esteves e Meiriño (2015, p. 2) afirmam:

O novo líder não se comporta mais como o controlador e sim como agente motivador envolvendo toda a organização, seja através de dinâmicas de grupo, reuniões, seminários, criando um ambiente de motivação e sensibilização, onde todos os envolvidos na estratégia organizacional compreendam a importância da educação continuada em conjunto com as metas empresariais.

A visão estratégica das empresas é melhor compreendida por um corpo docente formado por gestores, líderes e executivos de instituições corporativas, de modo a agregar valor à cadeia produtiva, empregando a própria força de trabalho da empresa para trazer benefícios ao conhecimento organizacional. Por esse e pelos seguintes motivos, a EC é diferente do ensino escolar tradicional (ESTEVES; MEIRIÑO, 2015):

a) $\mathrm{Na} \mathrm{EC}$, espaço físico é mais um conceito do que uma realidade;

b) O aprendizado através de ambientes virtuais (eletrônicos) na EC traz flexibilidade, havendo liberdade de escolha quanto ao melhor momento para as atividades;

c) O tempo menor de aprendizado em EC proporciona redução de custos;

d) O conhecimento vai ao encontro do indivíduo na EC, não havendo assim necessidade de ausentar-se; e 
e) Na EC, o público-alvo são os gestores/colaboradores, os clientes, os fornecedores e a comunidade em geral, cujo atendimento se dá através de ações calcadas na responsabilidade social e ambiental.

Devido às dificuldades de certificação de cursos (visto que tais certificações e diplomas só podem ser emitidos por órgãos credenciados pelo Ministério da Educação), as empresas têm investido nas parcerias com universidades públicas e privadas para validar certificações ou para elaborar cursos de EC . Devido ao envolvimento de indivíduos, grupos de trabalhos e até mesmo da comunidade na qual a empresa se situa, os programas de EC podem ser considerados um “processo evolutivo na área de Gestão de pessoas, visando a qualidade da força de trabalho, no gerenciamento estratégico da educação e treinamento" (ESTEVES; MEIRIÑO, 2015, p. 5).

Considerando as informações até aqui apresentadas, é possível discutilas e estabelecer entre si relações que viabilizem o entendimento sistematizado dos assuntos.

\section{Discussão}

Conhecimentos técnicos são importantes para o sucesso, mas competências necessárias aos cargos também são buscadas pelas empresas. Conhecimento tem prazo de validade e justamente por isso deve ser continuamente renovado, o que relaciona a carreira dos colaboradores às suas competências, habilidades, atitudes, vivências e buscas em prol do alcance de suas metas e objetivos estratégicos organizacionais (ESTEVES; MEIRIÑO, 2015).

Para garantir que as competências dos gestores e colaboradores estejam alinhadas com as metas das empresas, é necessário que a GC viabilize o fluxo de conhecimento entre os indivíduos e os grupos das organizações. Esse conhecimento deve ser adquirido pela empresa, identificado e codificado por pessoas responsáveis por seu armazenamento. Feito isto, o conhecimento pode então ser distribuído 
pelos diversos níveis das corporações, o que resulta na sua utilização para a consecução dos processos finalísticos das empresas.

A GC exige então que a empresa tenha a $\mathrm{AO}$ em perspectiva, pois é através da aprendizagem que os conhecimentos coletados e armazenados pela organização serão adaptados à sua cultura, disseminados entre seus colaboradores e gestores, adaptados e empregados na contínua melhoria das competências dos diversos indivíduos integrantes dos grupos de trabalho. Para que a AO seja uma realidade, exige-se que as organizações planejem e desenvolvam programas de EC, a fim de sistematizar e fomentar a cultura da aprendizagem contínua dos diversos stakeholders envolvidos na cadeia produtiva e nas práticas do negócio (colaboradores, fornecedores, clientes, comunidade e outros) (ESTEVES; MEIRIÑO, 2015).

Os estudos baseados em práticas organizacionais partem da noção de uma realidade emergente e do conhecimento como atividade material conectada a artefatos materiais. Sendo assim, o social não está relacionado somente a pessoas, mas também a artefatos simbólicos e culturais. A noção de prática articula a noção do espaço-tempo do fazer dos atores, ou seja, das práticas situadas nas empresas, o que implica incertezas e conflitos nas práticas de ensino e aprendizagem nas empresas (ANTONELLO; GODOY, 2009).

As novas noções implicadas no processo de AO trazem consideráveis compreensões sobre sua natureza, o que contribui com a compreensão da dinâmica das organizações e do fluxo de mudança desses sistemas sociais. Para que essas compreensões sejam obtidas, é necessário que os métodos de ensino e de aprendizagem sejam constantemente repensados, devido à natureza interpretativa e temporal dos processos de $\mathrm{GC}$ e $\mathrm{AO}$, os quais se traduzem no planejamento, no ensino, na aprendizagem e na avaliação de resultados dos programas de EC (ANTONELLO; GODOY, 2009).

A EC, por sua vez, pode contemplar diversas modalidades de ensino: cursos técnicos, educação básica, pós-graduação e outros. Em outros tempos, o processo de aprendizagem tinha começo, meio e fim, e quando o ciclo era 
completado, o indivíduo parava de aprender, já que o foco era desenvolver qualificações isoladas. No entanto, as organizações atualmente percebem a necessidade de constante renovação para fomentar uma cultura de contínuo desenvolvimento de pessoas, com qualificações mais amplas.

Este novo paradigma de aprendizagem contínua nas empresas, de comprometimento das organizações com a educação e o incremento de competências individuais garante que o compartilhamento de inovações corrobore com o alcance dos resultados delineados na estratégia empresarial (ESTEVES; MEIRIÑO, 2015), o que confere grande relevância à GC, à AO e à EC.

No panorama da atual GC, a $\mathrm{AO}$ é um processo baseado em competências, habilidades e atitudes. Capacidade humana e conhecimento, vitais para as empresas, são ativos intangíveis e não mensuráveis, embora produzam benefícios para as organizações. Deste modo, os programas de EC desenvolvidos nas corporações buscam a sobrevivência das organizações no mercado competitivo, agregando valor ao negócio e aplicando estratégias do conhecimento para aprimorar a eficiência operacional, alavancando as vantagens competitivas ao aprender mais rápido que as empresas concorrentes (ESTEVES; MEIRIÑO, 2015).

A EC deixa perceptível que o modelo de T\&D não mais satisfaz o desafio que as empresas encaram para desenvolver e reter capital intelectual, a fim de aumentar a competitividade na era do conhecimento e uso do conhecimento com foco em resultados, sendo, portanto, atribuição da gestão de pessoas a renovação na área de T\&D (ESTEVES; MEIRIÑO, 2015).

\section{Considerações Finais}

Os programas de EC nas organizações devem adotar mecanismos para avaliar e mensurar os resultados organizacionais, colocando em igual relevância da evolução e do desenvolvimento da estratégia organizacional a GC e a AO (ESTEVES; MEIRIÑO, 2015). Entretanto, ainda existem problemas quanto a 
regulamentação de cursos e emancipação de universidades corporativas, os quais ainda se configuram como dificuldades à prática da EC, havendo ainda espaço para o surgimento de novas modalidade de ensino e de parcerias para a consecução da aprendizagem contínua nas empresas.

Organizações que sustentam o processo de iniciação do conhecimento, que preparam o indivíduo para a aprendizagem e a reflexão sobre o mundo e sobre si mesmo devem ser reconhecidas como instituições basilares da EC, cujo processo se dá em função do envolvimento de gestores e líderes, agentes das mudanças provocadoras da constante busca do conhecimento e da evolução do capital humano, preenchendo a lacuna deixada pelo Estado na educação tradicional ao abranger a preparação/capacitação de profissionais para produção e desempenho superiores, consistentes com os objetivos das corporações (ESTEVES; MEIRIÑO, 2015).

Assim como são diversos os tipos de organizações, há diversos modelos de $\mathrm{EC}$ e $\mathrm{AO}$, os quais podem funcionar como repositórios da aprendizagem efetuada pelas empresas. Os diferentes modelos criam oportunidades que justificam que os responsáveis pela GC e os estudiosos das organizações corporativas, da comunicação e dos sistemas de informação tomem consciência dos trabalhos efetuados por pesquisadores de outras áreas de estudo, os quais podem apresentar novas soluções e abordagens para os desafios enfrentados pelas organizações (SANTANA, 2005).

As diversas organizações corporativas também podem se valer das investigações existentes sobre as modelagens de EC, a fim de refinar modelos em consonância com as próprias características institucionais e culturais. Esse refinamento da EC possibilita o desenvolvimento de novas e melhores ferramentas de análise intra e interorganizacional.

A disseminação e o compartilhamento do conhecimento entre as organizações podem aumentar exponencialmente os benefícios da GC, das práticas de AO e dos programas de EC. Esta possibilidade corrobora para que as boas práticas sejam mais ainda difundidas na sociedade, contribuindo para 
que novas pesquisas sejam desenvolvidas, para identificar novas oportunidades de carreira para as pessoas, trazer maiores benefícios sociais para as comunidades e potencializar o sucesso das empresas brasileiras.

\section{Referências}

ANTONELLO, C. S. GODOY, A. S. Uma agenda brasileira para os estudos em aprendizagem organizacional. RAE, São Paulo, v. 49, n. 3, jul./set. 2009, p. 266-281.

CRUZ, D. Educação corporativa: a proposta empresarial no discurso e na prática. Educação em Revista, Belo Horizonte, v. 26, n. 2, p. 337-358, ago. 2010.

ESTEVES, L. P. MEIRIÑO, M. J. A educação corporativa e a gestão do conhecimento. XI Congresso Nacional de Excelência em Gestão, ISSN 1984-9354, ago. 2015.

NEVES, P. C. CERDEIRA, J. P. Memória organizacional, gestão do conhecimento e comportamentos de cidadania organizacional. Perspectivas em Gestão \& Conhecimento, João Pessoa, v. 8, n. 1, p. 3-19, jan./abr. 2018.

PARÉ, G. et al. Synthesizing information systems knowledge: a typology of literature reviews. Information \& Management, vol. 52, n. 2, mar. 2015, p. 183-199.

PATON, R.; PETERS, G; QUINTAS, P. Estratégias de Educação Corporativa: universidades corporativas na prática. Ministério do Desenvolvimento, Indústria e Comércio Exterior, Brasília, 2007.

SANTANA, S. Modelo integrado para o estudo da aprendizagem organizacional. Análise Social, vol. XL (175), p. 367-391, 2005.

TOZONI-REIS, M. F. C. Metodologia da pesquisa. $2^{\mathrm{a}}$. ed. Curitiba: IESDE Brasil S.A., 2009. 